\title{
Domain-specific transition systems and their application to a formal definition of a model programming language*
}

\author{
I. S. Anureev
}

\begin{abstract}
The paper presents a new object model of domain-specific transition systems, a formalism designed for the specification and validation of formal methods for assuring software reliability. A formal definition of a model programming language is given on the basis of this model.
\end{abstract}

Keywords: state transition systems, domain-specific transition systems, operational semantics.

\section{Introduction}

Assuring software reliability is an urgent problem of the theory and practice of programming. Formal methods play an important role in solving this problem. Currently, there are quite a lot of reliable software development tools based on formal methods. They cover many aspects, from design and prototyping of software systems to their formal specification and verification.

However, while in the Semantic Web there is a tendency to integrate heterogeneous data and services, in the reliable software development we are still dealing with a set of separate tools, each of which covers only certain specific aspects of the development and, as a rule, is designed for use only with a small number of computer languages. The gap between the great potential of formal methods and, with a rare exception, toy examples of their application is also noticeable [11]. Among the obstacles that prevent a widespread introduction of formal methods to software development, we note the difficulties to master them, the high price of their introduction, and the fact that the software engineers and programmers are skeptical about them. Insufficient attention is also focused on the technological aspects of the development of formal semantics of computer languages, which plays an important role in assuring the software reliability.

A unified approach to assuring the software reliability which covers the stages of software development such as prototyping, design, specification, and verification of software systems was proposed in $[10,6,2]$. This approach was also used to develop a formal operational semantics and safety logic

\footnotetext{
*Partially supported by the RAS project N 15/10 and SB RAS interdisciplinary integration project $\mathrm{N} 3$.
} 
(a variant of axiomatic semantics) of computer languages [5]. It is based on a special kind of transition systems, domain-specific transition systems (DSTSs).

DSTSs can also be considered as "technological" abstract state machines [8], in which the rules for defining the states and the transition relation are explicitly specified. In this case, DSTSs provide a higher level of abstraction in specifying software systems in comparison with the implementation languages of abstract state machines ASML [7] and XASM [9].

The Atoment language for specification of DSTSs and the sublanguages for specification of particular kinds of DSTSs focused on solving the tasks of reliable software development were presented in $[6,4,3]$. The Atomentoriented object model of DSTSs was presented in [1]. In this paper, we describe the language-independent object model of DSTS and apply it to define formally a model programming language.

\section{Preliminaries}

Let int, nat, and bool denote the set of integers, the set of natural numbers, and the set $\{$ true, false , respectively.

Let $S e t^{*}$ denote the set of all finite sequences consisting of the elements of a set $S e t, S^{+} t^{+}$denote the set of all finite nonempty sequences consisting of the elements of Set, and pset $(S e t)$ denote the set of all subsets of Set. Let empseq denote the empty sequence, and $E l_{1} \ldots E l_{N}$ denote the sequence consisting of the elements $E l_{1}, \ldots, E l_{N}$. Let len $(S e q)$, Seq.I, first $(S e q)$, and last $(S e q)$ denote the length of a sequence $S e q$ and its $I$-th, first, and last elements, respectively.

Let $S e t \rightarrow S e t^{\prime}$ denote the set of all functions from $S e t$ to $S e t^{\prime}$, and Set $\rightarrow_{t} S e t^{\prime}$ denote the set of all total functions from Set to $S^{\prime} t^{\prime}$. Let $\operatorname{dom}($ Fun $)$ denote the domain of a function Fun, and und denote the indeterminate value. We assume that $F u n(\operatorname{Arg})=$ und, if $\operatorname{Arg} \notin \operatorname{dom}(F u n)$. Let $\operatorname{dom}($ Fun $) \cap \operatorname{dom}\left(F u n^{\prime}\right)=\emptyset$. The union Fun $\cup$ Fun ${ }^{\prime}$ of the functions Fun and Fun' is defined as the function Fun" such that $\operatorname{dom}\left(F u n^{\prime \prime}\right)=$ $\operatorname{dom}($ Fun $) \cup \operatorname{dom}\left(F u n^{\prime}\right), F_{u n}^{\prime \prime}(A r g)=F u n(A r g)$ for $\operatorname{Arg} \in \operatorname{dom}(F u n)$, and $F_{u n}^{\prime \prime}(A r g)=F u n^{\prime}(A r g)$ for $\operatorname{Arg} \in \operatorname{dom}\left(F u n^{\prime}\right)$. Let range(Fun) denote the range of Fun, i.e. the set $\{F u n(A r g) \mid \operatorname{Arg} \in \operatorname{dom}(F u n)\}$. Let $\operatorname{graph}(F u n)$ denote the graph of Fun, i.e. the set $\{(\operatorname{Arg}$, Fun $(\operatorname{Arg})) \mid \operatorname{Arg} \in \operatorname{dom}(F u n)\}$.

The boolean function odif is defined as follows: odif $\left(F u n, F u n^{\prime}, S e t\right)=$ true if and only if Fun $(A r g)=F u n^{\prime}(A r g)$ for $A r g \notin S e t$. Thus, the values of the functions Fun and Fun' may differ only at the elements of Set.

We say that Set is defined by the functions $F u n_{1}, \ldots, F u n_{N}$, if $\operatorname{dom}\left(F u n_{I}\right)=S e t$ for each $1 \leq I \leq N$, and information about Set is specified only by these functions. For simplicity, we will omit the only argument of these functions where it will not cause collisions. For example, we can write 
$F_{1} n_{1}$ instead of $F_{1}(E l)$ for some implicit argument $E l \in S e t$.

\section{The main concepts of the theory of domain-specific transition systems}

The set dsts of domain-specific transition systems is defined by the functions el, par, elgen, frm, match, and atom.

The set el(Dsts) of elements includes integers and boolean values, i.e. int $\cup$ bool $\subset$ el. The element sequences (in particular, elements as oneelement sequences) have static and dynamic semantics. The static semantics of Elseq defines the value $\operatorname{val}($ Elseq, St $) \in$ el returned by Elseq in the current state $S t$ of the system Dsts. The function val and the set st of states are defined below. In this case, Elseq can be considered as a query to $D s t s$ to get information about the current state $S t$ of the system Dsts. The dynamic semantics of Elseq defines how Elseq change the current state of $D s t s$, i.e. it defines the set of states to which Dsts can go from the current state by Elseq. In this case, Elseq can be considered as an instruction controlling the state of Dsts.

The set $\operatorname{par}(D s t s)$ of parameters, where $\operatorname{par}(D s t s) \subseteq \mathrm{el}$, is defined by the functions vkind, skind, and catched such that skind(Par) $\in$ $\{$ elem, seq $\}$, vkind $($ Par $) \in\{$ eval, quote $\}$, and catched(Par $) \in$ bool.

The parameters are used as the pattern parameters in the pattern matching. If skind $(P a r)=$ elem, the pattern matching associates Par with an element. If skind $(P a r)=$ seq, the pattern matching associates Par with (possibly empty) an element sequence. The function skind is called a parameter structure specifier.

The function catched specifies the propagation of the indeterminate value false in the definition of the function val (see below). The element false plays the role of both the boolean and the indeterminate value.

The element sequences associated with parameters are converted to the parameter values and used as arguments of the functions defining the static semantics of these element sequences. Let Par be associated with ElSeq. If vkind $(P a r)=$ eval, $P a r$ is called an evaluated parameter, and $\operatorname{val}(E l s e q, S t)$ is the value of $P a r$. If vkind $(P a r)=$ quote, $P a r$ is called a quoted parameter, and Elseq is the value of Par. The function vkind is called a parameter value specifier.

The set elgen(Dsts) of element generators is defined by the functions sem and embedded such that $\operatorname{sem}($ Elgen $)$ is a function, range $(\operatorname{sem}($ Elgen $)) \subseteq \mathrm{el}$, embedded(Elgen $) \in$ nat $\rightarrow$ bool, dom(embedded(Elgen $)) \subseteq$ $\{I \in$ nat $\mid 1 \leq I \leq \operatorname{arity}(\operatorname{sem}($ Elgen $))\}$, and if $\operatorname{Arg} \in \operatorname{sem}($ Elgen $)$, $1 \leq I \leq \operatorname{arity}(\operatorname{sem}(E l g e n))$, and embedded $($ Elgen $)(\operatorname{Arg} . I)=$ true, then Arg. $I \in \mathrm{el}^{*}$.

The element generators are used to generate new kinds of elements. The 
element $E l$ is generated by Elgen, if $E l=\operatorname{sem}(E l g e n)(A r g)$ for some $A r g \in$ $\operatorname{dom}(\operatorname{sem}($ Elgen $))$.

The element generators also define the embedded structure of element sequences. The element $E l^{\prime}$ appears in $E l$, if $E l^{\prime}=E l$, or $E l=$ $\operatorname{sem}(E l g e n)(A r g)$, and $E l^{\prime}$ appears in Arg.I for some $1 \leq I \leq \operatorname{len}($ Arg $)$ such that embedded $($ Elgen $)(I)=$ true.

The set elgen includes the object seqcomp such that sem(seqcomp) $\epsilon$ $\mathrm{el}^{*} \rightarrow_{t}$ el is a bijection, and embedded(seqcomp $)(1)=$ true. The object seqcomp is called a sequential element composition.

For simplicity, below we write Elgen instead of sem(Elgen). For example, we write seqcomp instead of sem(seqcomp).

The elements of the set sub $=\mathrm{el} \rightarrow \mathrm{el}^{*}$ are called substitutions. If $\operatorname{dom}(S u b)=\left\{X_{1}, \ldots, X_{n}\right\}, S u b$ can be written as $\left\{\left(X_{1} \leftarrow S u b\left(X_{1}\right)\right), \ldots\right.$, $\left.\left(X_{n} \leftarrow \operatorname{Sub}\left(X_{n}\right)\right)\right\}$. The substitution function subst $\in \mathrm{el}{ }^{*} \times \mathrm{sub} \rightarrow \mathrm{el}^{*}$ is defined as follows (the first proper rule is applied):

- $\operatorname{subst}($ empseq, $S u b)=$ empseq;

- if $E l \in \operatorname{dom}(S u b)$, then subst $(E l, S u b)=S u b(E l)$;

- $\operatorname{subst}(\operatorname{sem}(E l g e n)(A r g), S u b)=\operatorname{sem}(E l g e n)\left(A^{\prime} g^{\prime}\right) ;$

- $\operatorname{subst}(E l, S u b)=E l$;

- $\operatorname{subst}(E l E l s e q, S u b)=\operatorname{subst}(E l, S u b) \operatorname{subst}(E l s e q, S u b)$.

The sequence $A r g^{\prime}$ is defined as follows:

- if embedded $(E l g e n)(I)=$ true, then $\operatorname{Arg}^{\prime} . I=\operatorname{subst}(\operatorname{Arg}, S u b)$;

- if embedded $($ Elgen $)(I) \neq$ true, then Arg $^{\prime} . I=A r g$.

Substitutions are used to associate parameters with the element sequences as a result of the pattern matching, and to associate parameters with their values.

The set frm of forms is defined by the functions pat, pars, pcond, rvcond, and kind.

A form Frm defines the static and dynamic semantics for the set of element sequences called the instances of the form. The pattern matching uses the functions pat, pars, and pcond to define whether Elseq is an instance of Frm.

The sequence $\operatorname{pat}(\mathrm{Frm}) \in \mathrm{el}^{+}$is called a pattern of Frm.

The sequence $\operatorname{pars}(F r m) \in$ par* $^{*}$ such that the elements of $\operatorname{pars}(F r m)$ are pairwise distinct defines the parameters of the pattern pat $(F r m)$. Let $1 \leq I \leq \operatorname{len}(\operatorname{pars}(F r m))$. The element $\operatorname{par}(F r m) . I$ is called a parameter of Frm. The number len(pars $(F r m))$ is called the arity of Frm denoted by $\operatorname{arity}(F r m)$. 
A form defines the static semantics of its instances by its value. The value of $\mathrm{Frm}$ is defined as a function of the values of its parameters.

The element $\operatorname{pcond}(\mathrm{Frm}) \in \mathrm{el}$ is called a parameter condition of Frm. It defines a restriction on the values of the parameters of $F r m$. The sequence Elseq is an instance of Frm only if this restriction is satisfied.

The element $\mathrm{rvcond}(\mathrm{Frm}) \in \mathrm{el}$ is called a return-value condition of Frm. It defines a restriction on the value of Frm. The condition rvcond $(F r m)$ can include the parameters of Frm and the element retval $\in$ el, where retval $\in$ elgen, which refers to the value of Frm.

The partial function $\operatorname{kind}(F r m) \in$ \{statedependent, statefree, defined\} defines the kind of Frm. Thus, forms are divided into four kinds (the fourth kind corresponds to kind $(F r m)=$ und), and each kind has its own semantics.

The forms of the fourth kind define the state of Dsts. The function $S t \in$ $\{F r m \mid \operatorname{kind}(F r m)=$ und $\} \rightarrow \cup_{n \in \text { nato }}\left(\mathrm{el}^{n} \rightarrow_{t}\right.$ el $)$ such that $\operatorname{St}(F r m) \in$ $\mathrm{el}^{\operatorname{arity}(F r m)} \rightarrow_{t}$ el for all Frm is called the state of Dsts. Let st be the set of all states of Dsts. The state $S t$ is called empty, if $\operatorname{dom}(S t)=\emptyset$. The element $S t(F r m)$ is called the value of Frm in $S t$.

The form Frm of the kind statedependent is called a state-dependent predefined form. It is additionally defined by the function frmsem such that $\operatorname{frmsem}(F r m) \in \mathrm{st} \rightarrow \cup_{N \in \text { nato }}\left(\mathrm{el}^{N} \rightarrow_{t} \mathrm{el}\right)$, and $\operatorname{frmsem}(F r m)(S t) \in$ $\mathrm{el}{ }^{\operatorname{arity}(F r m)} \rightarrow_{t} \mathrm{el}$. The function frmsem is called a form semantics. The element $\operatorname{frmsem}(\mathrm{Frm})(\mathrm{St})$ is called the value of Frm in St.

The form Frm of the kind statefree is called a state-free predefined form. It is additionally defined by the function frmsem such that $\operatorname{frmsem}(F r m) \in \cup_{N \in \text { nato }}\left(\mathrm{el}^{N} \rightarrow_{t} \mathrm{el}\right)$, and $\operatorname{frmsem}(F r m) \in \mathrm{el}^{\operatorname{arity}(F r m)} \rightarrow_{t}$ el. The function frmsem is called a form semantics. The element frmsem $(F r m)$ is called the value of Frm in St.

The form Frm of the kind defined is called a defined form. It is additionally defined by the function body such that body $(F r m) \in \mathrm{el}^{+}$, which specifies the value of Frm. The elements of $\operatorname{body}(F r m)$ can include the parameters of Frm. Let $S u b^{\prime}$ map the parameters of Frm onto their values. The value of Frm in $S t$ is a function which maps the values of parameters of Frm, represented by $S u b^{\prime}$, onto val(subst(body $\left.\left.(F r m), S u b^{\prime}\right), S t\right)$. The function val is defined below.

The function $\operatorname{match}(D s t s) \in \mathrm{el}^{+} \rightarrow \operatorname{pset}(\mathrm{frm} \times \mathrm{sub})$ is called a form matching if for all $(F r m, S u b) \in \operatorname{match}(E l s e q)$ the following properties are satisfied:

- $E l s e q=\operatorname{subst}(F r m, S u b)$;

- $\operatorname{dom}(S u b)$ is the set of parameters of Frm;

- if $\operatorname{skind}(P a r)=$ elem, then $S u b(P a r) \in$ el; 
- if $\operatorname{skind}(P a r)=$ seq, then $S u b(P a r) \in$ el*;

- $\operatorname{arity}(F r m)=\operatorname{arity}\left(F r m^{\prime}\right)$, and $S u b(\operatorname{pars}(F r m) . I)=$ $S u b^{\prime}\left(\operatorname{pars}\left(F r m^{\prime}\right) . I\right)$ for all $\left(F r m^{\prime}, S u b^{\prime}\right) \in \operatorname{match}($ Elseq $)$, and $1 \leq$ $I \leq \operatorname{arity}(F r m)$.

The sequence Elseq is called an instance of Frm w.r.t. Sub, if $(F r m, S u b) \in$ match $(D s t s)(E l s e q)$ for some $S u b$. The sequence Elseq is called an instance of Frm, if Elseq is an instance of Frm w.r.t. some $S u b$.

The function match $(D s t s) \in \mathrm{el}^{+} \times \mathrm{st} \rightarrow \mathrm{frm} \times \mathrm{sub} \times \mathrm{sub}$ is called a form matching with parameter meaning if $\operatorname{match}($ Elseq, St $)=\left(F r m, S u b, S u b^{\prime}\right)$ if and only if the following properties are satisfied:

- $($ Frm,$S u b) \in \operatorname{match}($ Dsts $)($ Elseq $)$;

- $S u b^{\prime}=\operatorname{parval}(\operatorname{pars}(F r m), S u b, S t)$;

- $\operatorname{val}\left(\operatorname{subst}\left(\operatorname{pcond}(F r m), S u b^{\prime}\right), S t\right)=$ true.

It matches the form and the element sequence and sets the values of the parameters of this form. The function parval that sets the values of the parameters of the matched form is defined below.

The sequence Elseq is called an instance of Frm in $S t$ w.r.t. the matching substitution $S u b$ and the parameter meaning $S u b^{\prime}$, if $\operatorname{match}(E l s e q, S t)=$ $\left(F r m, S u b, S u b^{\prime}\right)$. The sequence Elseq is called an instance of Frm in $S t$, if Elseq is an instance of Frm in $S t$ w.r.t. some $S u b$ and $S u b^{\prime}$.

The set elgen(Dsts) includes the functions quote $\in \mathrm{el}^{+} \rightarrow \mathrm{el}$, and eval $\in \mathrm{el}^{+} \rightarrow$ el such that embedded(quote, 1$)=$ embedded(eval, 1$)=$ true. They specify the value of Par in the case when $S u b(P a r)$ has the form eval(Elseq) or quote(Elseq). If $S u b(P a r)=$ eval(Elseq), then $S u b^{\prime}(P a r)=\operatorname{val}(E l s e q, S t)$. If $S u b(P a r)=$ quote $(E l s e q)$, then $S u b^{\prime}(P a r)=$ Elseq.

The function parval $\in$ par $^{*} \times$ sub $\times$ st $\rightarrow$ sub sets the values of parameters in accordance with the element sequences which match these parameters:

- if $\operatorname{sub}($ Par $)=\operatorname{eval}($ Elseq $)$, then $\operatorname{parval}($ Par Parseq,Sub, St $)=$ $\{($ Par $\leftarrow \operatorname{val}($ Elseq, St $))\} \cup \operatorname{parval}($ Parseq, Sub,$S t)$;

- if $\operatorname{sub}($ Par $)=$ quote $($ Elseq $)$, then parval $($ Par Parseq, Sub, St $)=$ $\{($ Par $\leftarrow$ Elseq $)\} \cup \operatorname{parval}($ Parseq, Sub,$S t)$;

- if $\operatorname{vkind}(P a r)=$ eval, and skind $(P a r)=$ elem, then parval $(P a r$ Parseq, Sub,$S t)=\{($ Par $\leftarrow \operatorname{val}($ Sub $($ Par $), S t))\} \cup \operatorname{parval}($ Parseq, Sub, St);

- if $\operatorname{vkind}(P a r)=$ eval, $\operatorname{skind}(P a r)=$ seq, and $S u b(P a r)=$ Elorpar $_{1} \ldots$ Elorpar $_{N}$, then parval $($ Par Parseq,Sub,St $)=$ $\left\{\left(\right.\right.$ Par $\leftarrow$ ifval $\left(\right.$ Elorpar $_{1}$, eval, St $) \ldots$ ifval $\left(\right.$ Elorpar $_{N}$, eval, $\left.\left.\left.S t\right)\right)\right\}$ $\cup$ parval $($ Parseq, Sub, St); 
- if $\operatorname{vkind}(P a r)=$ quote, and skind $(P a r)=$ elem, then parval(Par Parseq, Sub, St $)=\{($ Par $\leftarrow$ Sub(Par $))\} \cup \operatorname{parval}($ Parseq, Sub, St);

- if $\operatorname{vkind}(P a r)=$ quote, $\operatorname{skind}(P a r)=$ seq, and $S u b(P a r)=$

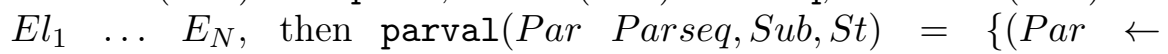
ifval $\left(E l_{1}\right.$, quote, $\left.S t\right) \ldots$ ifval $\left(E l_{N}\right.$, quote, $\left.\left.\left.S t\right)\right)\right\} \cup$ parval(Parseq, $S u b, S t)$.

The function ifval $\in \mathrm{el}^{+} \times$st $\times\{$eval, quote $\} \rightarrow \mathrm{el}$ is defined as follows:

- ifval (eval (Elseq), St, Vparorqpar $)=\operatorname{val}($ Elseq, St $)$;

- ifval(quote(Elseq), St, Vparorqpar) = Elseq;

- ifval $(E l, S t$, eval $)=\operatorname{val}(E l, S t)$;

- ifval $(E l, S t$, quote $)=E l$.

The function val $\in \mathrm{el}^{+} \times \mathrm{st} \rightarrow \mathrm{el}$ called an element sequence meaning is defined as follows (the first proper rule is applied):

- $\operatorname{val}($ true,$S t)=$ true;

- if $\operatorname{match}(E l s e q)=\left(F r m, S u b, S u b^{\prime}\right), \operatorname{pars}(F r m)=\operatorname{Par}_{1} \ldots \operatorname{Par}_{N}$, $A r g=S u b^{\prime}\left(\operatorname{Par}_{1}\right), \ldots, S u b^{\prime}\left(\operatorname{Par}_{N}\right)$, and Retvalcond $(U)$ denotes

$\operatorname{val}\left(\operatorname{subst}\left(\operatorname{rvcond}(F r m), S u b^{\prime} \cup\{(\operatorname{retval}(D s t s) \leftarrow U)\}\right), S t\right)=$ true,

then

- if catched $\left(\operatorname{Par}_{I}\right) \neq$ true, and $S u b^{\prime}\left(\operatorname{Par}_{I}\right)=$ false for some $1 \leq I \leq \operatorname{arity}(F r m)$, then $\operatorname{val}(E l s e q, S t)=$ false;

- if $\operatorname{kind}(F r m)=$ und, $S t(F r m) \neq$ und, and Retvalcond $(S t($ Frm $)(A r g))$, then val $($ Elseq, St $)=S t(F r m)(A r g)$;

- if $\operatorname{kind}(F r m)=$ statedependent, and Retvalcond(frmsem $(F r m$ )$(S t)(A r g))$, then val $($ Elseq, $S t)=f \operatorname{rrmsem}(F r m)(S t)(A r g)$;

- if $\operatorname{kind}(F r m)=$ statefree, and Retvalcond $(\operatorname{frmsem}(\mathrm{Frm})($ $A r g)$ ), then val $($ Elseq, St $)=\operatorname{frmsem}(F r m)(A r g)$;

- if kind $(F r m)=$ defined, and Retvalcond(val(subst(body ( $\left.\left.\left.F r m), S u b^{\prime}\right), S t\right)\right)$, then $\operatorname{val}($ Elseq, $S t)=\operatorname{val}(\operatorname{subst}(\operatorname{body}(F r m$ ), $\left.\left.S u b^{\prime}\right), S t\right)$;

- if $\operatorname{atom}(D s t s)(E l s e q)=$ true, then $\operatorname{val}(E l s e q, S t)=E l s e q$;

- $\operatorname{val}($ Elseq,$S t)=$ false.

The element val $(E l s e q, S t)$ is called the value of Elseq in St.

The function atom $(D s t s) \in \mathrm{el}^{+} \rightarrow_{t}$ bool defines the element sequences which coincide with their values. Such sequences are called atoms. 
The dynamic semantics of the element sequences is defined by the function $\operatorname{tr} \in \operatorname{conf} \times \operatorname{conf} \rightarrow$ bool called a transition relation. The set conf of configurations and the function $\operatorname{tr}$ are defined below. The system Dsts can go from Conf to Conf $f^{\prime}$ if and only if $\operatorname{tr}\left(\operatorname{Conf}\right.$, Conf $\left.^{\prime}\right)=$ true.

The set conf of configurations is defined by the functions seq and st such that $\operatorname{seq}($ Con $f) \in \mathrm{el}^{*}$, and $\operatorname{st}($ Conf $) \in$ st. The sequence seq $($ Conf $)$ is called a control sequence of Conf. It defines the states to which Dsts can go from the current state and the control sequences executed in these states.

The configuration Conf is called a final one, if there is no configuration Conf $f^{\prime}$ such that $\operatorname{tr}\left(\right.$ Conf, Conf $\left.^{\prime}\right)=$ true. The sequence Conf seq $\in$ conf $^{+}$ is called a run, if last (Confseq) is a final configuration.

A final configuration Conf is called unsafe, if $\operatorname{seq}(\operatorname{Conf}) \neq$ empseq. It specifies incorrect termination of Dsts. A final configuration Conf is called safe, if Conf is not unsafe. A run Confseq such that last(Confseq) is unsafe, is called unsafe. A run Confseq is called safe, if Confseq is not unsafe. A configuration Conf is called unsafe, if there is an unsafe run Confseq such that first $($ Confseq $)=$ Conf. A configuration Conf is called safe, if Conf is not unsafe.

A sequence Elseq is correct in St, if Conf is safe, where $\operatorname{seq}($ Conf $)=$ Elseq, and st $($ Conf $)=S t$. A sequence Elseq is incorrect in St, if Elseq is not corrrect in $S t$.

The set elgen includes the function fail $\in$ el such that if first $(\operatorname{seq}(\operatorname{Con} f))=$ fail, then Conf is final. The configuration Conf is also unsafe, since seq $($ Con $f) \neq$ empseq. Therefore the element fail is called an unsafe termination.

Let $\operatorname{tr}\left(\right.$ Conf, Conf $\left.^{\prime}\right)=$ true. Then $\operatorname{seq}($ Conf $)$ and $\operatorname{seq}\left(\right.$ Conf $\left.^{\prime}\right)$ are called the input and output control sequences of the transition, and st $($ Conf $)$ and st $\left(\right.$ Conf $\left.^{\prime}\right)$ are called the input and output states of the transition, respectively.

The function tr is defined by a special kind of forms, or transition rules. A form Frm is called a transition rule, if it is additionally defined by the function rkind such that $r k i n d(F r m) \in\{$ defined, predefined $\}$. The function kind defines the kind of the rule. Thus, if $\operatorname{rkind}(F r m)=$ und, then Frm is not a transition rule, and transition rules are divided into two kinds, and each kind has its own semantics. Let rul(Dsts) be a set of all rules of Dsts, and Rul $\in \operatorname{rul}(D s t s)$.

A rule $R u l$ of the kind defined is called defined. It is additionally defined by the function body such that $\operatorname{body}(R u l) \in \mathrm{el}^{*}$. The sequence $\operatorname{body}(R u l)$ is called the body of $R u l$ and it defines the execution of $R u l$.

A rule $R u l$ of the kind predefined is called predefined. It is additionally defined by the function rulsem such that $\operatorname{rulsem}(R u l) \in \operatorname{conf} \times \operatorname{conf} \times$ sub $\rightarrow_{t}$ bool. This function is called a rule semantics and it defines the execution of Rul. The third argument of the function stores the values of 
the parameters of $R u l$.

The function $\operatorname{tr}$ is defined as follows: $\operatorname{tr}\left(\operatorname{Conf}, \operatorname{Conf}^{\prime}\right)=\operatorname{true}$ if and only if there is a rule $R u l$ such that $\operatorname{tr}\left(\operatorname{Conf}, C_{\left.\text {on } f^{\prime}, R u l\right)}=\right.$ true.

The function $\mathrm{tr}$ with an additional argument $R u l$ is defined as follows: $\operatorname{tr}\left(\right.$ Conf $\left., C o n f^{\prime}, R u l\right)=$ true if and only if seq $($ Conf $)=E l s e q E l s e q$, $\operatorname{match}(E l s e q, \operatorname{st}(C o n f))=\left(R u l, S u b, S u b^{\prime}\right)$, and one of two conditions is satisfied: $\operatorname{rkind}(R u l)=$ predefined, and $\operatorname{rulsem}(R u l)\left(\right.$ Conf,$C_{\text {onf }}^{\prime}$, $\left.S u b^{\prime}\right)=$ true, or $\operatorname{rkind}(R u l)=\operatorname{defined}, \operatorname{seq}\left(C_{o n f}\right)=\operatorname{subst}(\operatorname{body}(R u l)$, $\left.S u b^{\prime}\right) E_{s e q}$, and st $\left(C_{\text {Onf }}^{\prime}\right)=\operatorname{st}($ Conf $)$.

Thus, when a defined rule $R u l$ is applied, the state of Dsts does not change, and the control sequence changes only its prefix matched with $R u l$.

The configuration Conf is called final w.r.t. Rul, if there is no configuration Conf $^{\prime}$ such that $\operatorname{tr}\left(\operatorname{Conf}\right.$, Conf $\left.^{\prime}, R u l\right)=$ true.

\section{Domain-specific transition systems with backtracking}

The use of backtracking in DSTSs expands their expressive power.

A DSTS Dsts is called a DSTS with backtracking if the following properties are satisfied:

- conf is additionally defined by the function rulset such that $\operatorname{rulset}($ Conf $) \subseteq \operatorname{rul}($ Dsts $)$. This function specifies which transition rules have been applied in the transitions from the configuration Conf .

- Dsts is additionally defined by the function backfrm such that backfrm $(D s t s) \subseteq F r m$. The set backfrm $(D s t s)$ specifies the forms whose values are preserved when $D$ sts backtracks to the previous backtracking point.

The function ifst $\left(S t, S t^{\prime}\right)$ returns a state; it is defined as follows:

- if $F r m \in$ backfrm, then ifst $\left(S t, S t^{\prime}\right)(F r m)=S t^{\prime}(F r m)$;

- if Frm $\notin$ backfrm, then ifst $\left(S t, S t^{\prime}\right)(F r m)=S t(F r m)$.

DSTS with controlled backtracking. A DSTS Dsts with backtracking is called a DSTS with controlled backtracking, if

- elgen(Dsts) includes the functions backtrack and branch such that backtrack $\in \mathrm{el}$, branch $\in \mathrm{el}^{* *} \rightarrow_{t} \mathrm{el}$, and embedded(branch)(1) $=$ true. The element backtrack called a backtracking condition initiates backtracking to the previous backtracking point. The element $\operatorname{branch}(E l S e q S e q)$ called a branch element is used to define possible variants in the backtracking point given by the elements of ElSeqSeq.

- $\operatorname{tr} \in \operatorname{conf}^{*} \times \operatorname{conf}^{*} \rightarrow$ bool is a controlled backtracking. 
Let $\mathrm{ba}(S t)$ and $\mathrm{fi}(S t)$ be configurations such that seq $(\mathrm{ba}(S t))=$ backtrack, st $(\mathrm{ba}(S t))=S t, \operatorname{rulset}(\mathrm{ba}(S t))=\emptyset$, seq $(\mathrm{fi}(S t))=$ empseq, $\operatorname{st}(\mathrm{fi}(S t))=S t$, and $\operatorname{rulset}(\mathrm{ba}(S t))=\emptyset$.

A transition relation $\operatorname{tr} \in$ conf $^{*} \times$ conf $^{*} \rightarrow$ bool is called a controlled backtracking, if $\operatorname{tr}($ Confseq, Conf seq $)=$ true if and only if the first proper property is satisfied:

- Confseq $=$ Confseq" Conf, $\operatorname{seq}($ Conf $)=$ backtrack Elseq, where Elseq $\neq$ empseq, or rulset $($ Conf $) \neq \emptyset$, and Conf seq $=$ Confseq ba $($ st $($ Conf $))$;

- Confseq = Confseq" Conf ba $(S t)$, where $\operatorname{seq}($ Conf $)=$ branch (Elseq' Elseqseq) Elseq, Confseq' $=$ Confseq" Conf' Conf", $\operatorname{seq}\left(\right.$ Conf $\left.^{\prime}\right)=\operatorname{branch}($ Elseqseq $)$ Elseq, st $\left(\right.$ Conf $\left.^{\prime}\right)=\operatorname{st}($ Conf $)$, $\operatorname{rulset}\left(\right.$ Conf $\left.^{\prime}\right)=\operatorname{rulset}($ Conf $), \operatorname{seq}\left(\right.$ Conf $\left.^{\prime \prime}\right)=$ Elseq $q^{\prime}$ Elseq, $\operatorname{st}\left(\operatorname{Conf}^{\prime \prime}\right)=\operatorname{ifst}(\operatorname{st}(\operatorname{Conf}), S t)$, and $\operatorname{rulset}\left(\operatorname{Conf}^{\prime \prime}\right)=\emptyset$;

- Confseq $=$ Confseq" Conf' Conf ba(St), where $\operatorname{seq}($ Conf $)=$ branch(empseq) Elseq, and Conf seq ${ }^{\prime}=$ Confseq Conf $^{\prime}$ ba $(S t)$;

- Confseq $=$ Conf $\operatorname{ba}(S t)$, where $\operatorname{seq}\left(C_{\text {onf }}^{\prime}\right)=\operatorname{branch}($ empseq $)$ Elseq, and Confseq ${ }^{\prime}=\operatorname{ba}($ ifst $(\operatorname{st}(C o n f), S t))$;

- Confseq = Confseq" Conf, where seq(Conf) = branch (Elseq' Elseqseq) Elseq, Confseq ${ }^{\prime}=$ Confseq" Conf' $f^{\prime}$ Conf", $\operatorname{seq}\left(\right.$ Conf $\left.^{\prime}\right)=\operatorname{branch}($ Elseqseq $)$ Elseq, st $\left(\right.$ Conf $\left.^{\prime}\right)=\operatorname{st}($ Conf $)$, $\operatorname{rulset}\left(\right.$ Conf $\left.^{\prime}\right)=\operatorname{rulset}($ Conf $), \operatorname{seq}\left(\right.$ Conf $\left.^{\prime \prime}\right)=$ Elseq ${ }^{\prime}$ Elseq, $\operatorname{st}\left(\right.$ Conf $\left.^{\prime \prime}\right)=\operatorname{st}($ Conf $)$, and $\operatorname{rulset}\left(\right.$ Conf $\left.^{\prime \prime}\right)=\emptyset$;

- Confseq $=$ Confseq" Conf, where $\operatorname{seq}($ Conf $)=\operatorname{branch}($ empseq $)$ Elseq, and Confseq ${ }^{\prime}=$ Confseq" fi(st $($ Conf $\left.)\right)$;

- Confseq $=$ Confseq" Conf ba(St), where seq $($ Conf $)=E l s e q^{0}$ Elseq, Elseq ${ }^{0} \notin \operatorname{predel}(D s t s), R u l \in \operatorname{rul}(D s t s) \backslash \operatorname{rulset}(C o n f)$, $\operatorname{tr}\left(\right.$ Conf $^{2}$, Conf $^{3}$, Rul $)=$ true, $\operatorname{seq}\left(\right.$ Conf $\left.^{2}\right)=E_{\text {seq }}{ }^{0}$ Elseq, $\operatorname{st}\left(C_{\text {onf }}^{2}\right)=\operatorname{ifst}(\operatorname{st}($ Conf $), S t), \operatorname{seq}\left(C o n f^{3}\right)=E l s e q^{\prime}$, Conf seq ${ }^{\prime}=$ Confseq" Conf $^{4}$ Conf $^{5}, \operatorname{seq}\left(\right.$ Conf $\left.^{4}\right)=E l s e q^{0}$ Elseq, st $\left(\right.$ Conf $\left.^{4}\right)=$ $\operatorname{st}(\operatorname{Conf}), \operatorname{rulset}\left(\operatorname{Conf} f^{4}\right)=\operatorname{rulset}(\operatorname{Con} f) \cup\{R u l\}, \operatorname{seq}\left(\operatorname{Con} f^{5}\right)=$ $E l s e q^{\prime}, \operatorname{st}\left(C_{\text {onf }}^{5}\right)=\operatorname{st}\left(\right.$ Conf $\left.^{3}\right)$, and $\operatorname{rulset}\left(C_{\text {onf }}{ }^{5}\right)=\emptyset$;

- Confseq = Confseq" Conf' Conf ba(St), and Confseq $=$ Conf seq" Conf' ba $(S t)$;

- Confseq $=$ Conf ba(St), and Confseq $=$ ba(ifst(st(Conf), St));

- Confseq $=\mathrm{ba}(S t)$, and Confseq $=\mathrm{fi}(S t)$;

- Confseq = Confseq" Conf, where seq(Conf $)=E^{\prime \prime}$ lseq ${ }^{0}$ Elseq, $E l s e q^{0} \notin \operatorname{predel}, R u l \in \operatorname{rul}(D s t s) \backslash \operatorname{rulset}(\operatorname{Conf}), \operatorname{tr}\left(\right.$ Conf, Conf $f^{\prime}$, Rul $)=$ true, Confseq ${ }^{\prime}=$ Confseq ' Conf $^{\prime \prime}$ Conf $f^{\prime \prime \prime}$, seq $\left(\right.$ Conf $\left.f^{\prime \prime}\right)=$ 
$\operatorname{seq}($ Conf $), \quad \operatorname{st}\left(\right.$ Conf $\left.^{\prime \prime}\right)=\operatorname{st}($ Conf $), \operatorname{rulset}\left(\right.$ Conf $\left.^{\prime \prime}\right)=$ $\operatorname{rulset}($ Conf $) \cup\{R u l\}, \operatorname{seq}\left(\right.$ Con $\left.^{\prime \prime \prime}\right)=\operatorname{seq}\left(\right.$ Conf $\left.^{\prime}\right)$, st $\left(\right.$ Conf $\left.f^{\prime \prime \prime}\right)=$ $\operatorname{st}\left(\right.$ Conf $\left.^{\prime}\right)$, an rulset $\left(\right.$ Conf $\left.f^{\prime \prime \prime}\right)=\emptyset$;

- Confseq = Confseq" Conf, where seq(Conf $)=$ Elseq $q^{0}$ Elseq, Elseq $^{0} \in \operatorname{predel}($ Dsts $), \operatorname{rulsem}($ Dsts $)\left(\right.$ Conf, Conf $\left.f^{\prime}\right)=$ true, Confseq $=$ Confseq" Conf' Conf $^{\prime \prime \prime}, \operatorname{seq}\left(\right.$ Conf $\left.^{\prime \prime}\right)=$ Elseq, $\operatorname{st}\left(\right.$ Conf $\left.^{\prime \prime}\right)=\operatorname{st}($ Conf $), \quad \operatorname{rulset}\left(\right.$ Conf $\left.^{\prime \prime}\right)=\operatorname{rulset}($ Conf $)$, $\operatorname{seq}\left(\right.$ Conf $\left.^{\prime \prime \prime}\right)=\operatorname{seq}\left(\right.$ Conf $\left.^{\prime}\right)$, st $\left(\right.$ Conf $\left.^{\prime \prime \prime}\right)=\operatorname{st}\left(\right.$ Conf $\left.^{\prime}\right)$, and $\operatorname{rulset}\left(\right.$ Conf $\left.^{\prime \prime \prime}\right)=\emptyset$;

- false.

\section{Examples of predefined transition rules}

Let us consider examples of predefined transition rules which are often used to define operational semantics of computer languages.

Let elgen $(D s t s)$ include the function stop $\in$ el. A form $R u l$ is called a stop rule, if pat $(R u l)=$ stop, $\operatorname{arity}(R u l)=0, \operatorname{cond}(R u l)=$ true, $\operatorname{rkind}(R u l)=$ predefined, and $\operatorname{rulsem}(R u l)\left(\operatorname{Conf}, C_{\text {onf }}^{\prime}\right)=$ true, where $\operatorname{seq}($ Conf $)=$ stop Elseq, if and only if $\operatorname{seq}\left(C_{\text {onf }}^{\prime}\right)=$ empseq, and st $\left(\right.$ Conf $\left.^{\prime}\right)=\operatorname{st}($ Conf $)$. The element stop is called a stop element.

Let elgen (Dsts) include the function assume $\in \mathrm{el} \rightarrow \mathrm{el}$. A form Rul is called a continuation rule, if pat $(R u l)=$ assume $(\operatorname{Par}), \operatorname{pars}(R u l)=$ Par, $\operatorname{vkind}(P a r)=$ eval, $\operatorname{skind}(P a r)=$ seq, $\operatorname{cond}(R u l)=$ true, $\operatorname{rkind}(R u l)=$ predefined, and rulsem $(R u l)\left(\right.$ Conf, Con $\left.f^{\prime}\right)=$ true, where seq $($ Conf $)=$ El Elseq, if and only if $\operatorname{match}(E l$, st $($ Conf $))=\left(R u l, S u b, S u b^{\prime}\right)$, st $\left(C_{o n f}^{\prime}\right)=\operatorname{st}(C o n f)$, and the first proper property is satisfied:

- if $S u b^{\prime}(P a r)=$ true, then seq $\left(\right.$ Conf $\left.^{\prime}\right)=$ Elseq;

- $\operatorname{seq}\left(\right.$ Con $\left.^{\prime}\right)=$ backtrack Elseq.

The element $E l$ is called a continuation condition. This condition is based on the element backtrack and used in DSTS with controlled backtracking.

Let elgen(Dsts) include the function frmupd $\in \mathrm{el}^{+} \times \mathrm{el}^{+} \rightarrow \mathrm{el}$. A form $R u l$ is called a form update rule, if pat $(R u l)=\operatorname{frmupd}\left(\operatorname{Par}_{,} \operatorname{Par}^{\prime}\right)$, $\operatorname{pars}(R u l)=\operatorname{Par} P a r^{\prime}, \operatorname{vkind}(P a r)=$ quote, $\operatorname{vkind}\left(\operatorname{Par}^{\prime}\right)=$ eval, $\operatorname{skind}(P a r)=\operatorname{skind}\left(P a r^{\prime}\right)=\operatorname{seq}, \operatorname{cond}(R u l)=\operatorname{true}, \operatorname{rkind}(R u l)=$ predefined, and rulsem $(R u l)\left(\right.$ Conf, Conf $\left.f^{\prime}\right)=$ true, where seq $($ Conf $)=$ El Elseq, if and only if $\operatorname{match}(E l, \operatorname{st}(\operatorname{Conf}))=\left(R u l, S u b, S u b^{\prime}\right)$, and the first proper property is satisfied:

- if $\operatorname{match}\left(S u b^{\prime}(P a r), \operatorname{st}(C o n f)\right)=\left(F r m, S u b_{1}, S u b_{1}^{\prime}\right), \operatorname{kind}(F r m)=$ und, $\operatorname{arity}(F r m)=N, \operatorname{Arg}=S u b_{1}^{\prime}(\operatorname{pars}(F r m) .1), \ldots$, $S u b_{1}^{\prime}(\operatorname{pars}(F r m) . N)$, then seq $\left(\right.$ Conf $\left.^{\prime}\right)=E l s e q, \operatorname{odif}\left(\operatorname{st}\left(\right.\right.$ Conf $\left.^{\prime}\right)$, 
st $($ Conf $),\{F r m\})=\operatorname{true}, \operatorname{odif}\left(\operatorname{st}\left(C o n f^{\prime}\right)(F r m)\right.$, st $($ Conf $)(F r m)$, $\{($ Arg $)\})=$ true, and st $\left(\right.$ Conf $\left.^{\prime}\right)($ Frm $)(\operatorname{Arg})=S u b^{\prime}\left(\operatorname{Par}^{\prime}\right)$;

- $\operatorname{seq}\left(\right.$ Conf $\left.^{\prime}\right)=$ fail El Elseq, and $\operatorname{st}\left(\right.$ Conf $\left.^{\prime}\right)=\operatorname{st}($ Conf $)$.

The element $E l$ is called a form update.

Let elgen $(D s t s)$ include the function assert $\in \mathrm{el}^{+} \rightarrow \mathrm{el}$. A form $R u l$ is called a safety rule, if pat $(R u l)=\operatorname{assert}(\operatorname{Par}), \operatorname{pars}(R u l)=P a r$, $\operatorname{vkind}(P a r)=$ eval, $\operatorname{skind}($ Par $)=$ seq, $\operatorname{cond}(R u l)=$ true, $\operatorname{rkind}(R u l)=$ predefined, $\operatorname{rulsem}(R u l)\left(\right.$ Conf, Conf $\left.f^{\prime}\right)=$ true, where $\operatorname{seq}($ Conf $)=E l$ Elseq, if and only if match $(E l, \operatorname{st}($ Conf $))=\left(R u l, S u b, S u b^{\prime}\right)$, st $\left(C_{\text {onf }}^{\prime}\right)=$ st $(C o n f)$, and the first proper property is satisfied:

- if $S u b^{\prime}($ Par $)=$ true, then seq $\left(\right.$ Conf $\left.^{\prime}\right)=$ Elseq;

- $\operatorname{seq}\left(\right.$ Con $\left.^{\prime}\right)=$ fail El Elseq.

The element $E l$ is called a safety condition.

Let elgen $(D s t s)$ include the function cases $\in \operatorname{par} \times \operatorname{par} \cup \operatorname{par} \times \operatorname{par} \times$ par $\cup \mathrm{el}^{*} \times \mathrm{el}^{* *} \cup \mathrm{el}{ }^{*} \times \mathrm{el}^{* *} \times \mathrm{el}^{*} \rightarrow \mathrm{el}$, such that (Elseq, Elseqseq[, Elseq $\left.\left.q^{\prime}\right]\right) \in \operatorname{dom}($ cases $)$ if and only if $\operatorname{len}($ Elseq $)=\operatorname{len}($ Elseqseq $)$.

A form Rul is called a conditional branching rule, if pat $(R u l)=$ $\operatorname{cases}\left(\operatorname{Par} \operatorname{Par}^{\prime}\left[, \operatorname{Par}^{\prime \prime}\right]\right), \operatorname{pars}(R u l)=$ Par $\operatorname{Par}^{\prime} \operatorname{Par}^{\prime \prime}, \operatorname{vkind}(\operatorname{Par})=$ quote, and skind $(P a r)=$ seq for each $P a r \in \operatorname{pars}(R u l)$, $\operatorname{cond}(R u l)=$ true, $\operatorname{rkind}(R u l)=\operatorname{predefined}, \operatorname{rulsem}(R u l)\left(\right.$ Conf, Conf $\left.f^{\prime}\right)=$ true, where seq $($ Conf $)=E l$ Elseq, if and only if $\operatorname{match}(E l$, st $($ Conf $))=$ $\left(R u l, S u b, S u b^{\prime}\right), \quad \operatorname{st}\left(C_{o n f}\right)=\operatorname{st}($ Conf $), \quad$ and $\operatorname{seq}\left(C_{\text {Conf }}^{\prime}\right)=$ $\operatorname{branch}\left(\operatorname{Arg} .1, \ldots, \operatorname{Arg} . N \quad\left[, S u b^{\prime}\left(\operatorname{Par}^{\prime \prime}\right)\right]\right)$, where Arg.I = assume $\left(S u b^{\prime}(P a r) .1\right) S u b^{\prime}\left(P^{\prime} r^{\prime}\right) .1$ for $1 \leq I \leq \operatorname{len}\left(S u b^{\prime}(P a r)\right)=N$. The element $E l$ is called a conditional branching.

\section{Formal definition of the model programming language}

Let us define a simple model programming language MPL by DSTS.

The MPL language includes the set $i d$ of identifiers (sequences of letters from $\{\mathrm{a}, \ldots, \mathrm{z}, \mathrm{A}, \ldots, \mathrm{Z}\}$, digits from $\{0, \ldots, 9\}$, and the underscore character _, starting with a letter), the finite set btype $\subset$ id of basic types such that lit(Btype) is a set of literals of the type Btype $\in$ btype, lit (Btype) $\cap$ id $=\emptyset$ for each Btype, int $\in$ btype, $\operatorname{lit}($ int $)=\{\ldots,-2,-1,0,1,2, \ldots\}$, bool $\in$ btype, and $\operatorname{lit}($ bool $)=\{$ true, false $\}$, the operations $=$, and $!=$ on these types, the arithmetic operations,,$+- *$, div, mod, and the arithmetic relations $<,>,<=,>=$ on integers, the boolean operations and, or, not, implies, variable declaration, assignment statement, if statement, and while statement.

Let us consider Dsts which specifies MPL. The functions el, par, elgen, frm, match, and atom are defined as follows: 
- el $\stackrel{\text { def }}{=} \mathrm{id} \cup \cup_{\text {Elgen } \in \text { elgen }(D s t s)}$ range $($ Elgen $)$;

- $\operatorname{par}(D s t s)(E l)=$ true if and only if $E l \in$ id $\backslash$ dtype;

- elgen $(D s t s) \stackrel{\text { def }}{=}$ \{delcomp, eval, quote, fail, backtrack, branch, stop, assume, frmupd, assert, cases $\}$;

- if $\operatorname{len}($ Elseq $)=N$, then $\operatorname{delcomp}($ Elseq $) \stackrel{\text { def }}{=}($ Elseq.1 ... Elseq.N);

- eval(Elseq) $\stackrel{\text { def }}{=}($ eval Elseq);

- quote $(E l s e q) \stackrel{\text { def }}{=}$ (quote Elseq);

- $\operatorname{retval}(D s t s) \stackrel{\text { def }}{=}$ retval;

- fail $\stackrel{\text { def }}{=}$ fail;

- backtrack $\stackrel{\text { def }}{=}$ backtrack;

- $\operatorname{branch}($ Elseqseq $) \stackrel{\text { def }}{=}$ (branch Elseqseq);

- stop $\stackrel{\text { def }}{=}$ stop;

- assume $(E l s e q) \stackrel{\text { def }}{=}$ (assume Elseq);

- $\operatorname{frmupd}\left(E l s e q, E l s e q^{\prime}\right) \stackrel{\text { def }}{=}\left(E l s e q \quad::=E l s e q^{\prime}\right)$;

- $\operatorname{assert}(E l s e q) \stackrel{\text { def }}{=}$ (assert Elseq);

- if $\operatorname{len}($ Elseq $)=N$, then cases $($ Elseq, Elseqseq, Elseq $) \stackrel{\text { def }}{=}$ (cases (if Elseq.1 then Elseqseq.1) ...(if Elseq. $N$ then Elseqseq. $N)($ else Elseq $)$ );

- $\operatorname{frm}(D s t s) \stackrel{\text { def }}{=}\left\{F r m \_I d \mid I d \in \mathrm{id}\right\} \cup\left\{R u l_{-} I d \mid I d \in \mathrm{id}\right\}$;

- the algorithm match $(D s t s)$ choose the first proper element sequence from left to right. For example, if pat $(F r m)=($ if $X$ then $Y$ else $Z)$, $\operatorname{pars}(F r m)=X Y Z$, skind $(X)=$ elem, $\operatorname{skind}(Y)=$ seq, and $\operatorname{skind}(Z)=$ seq, then $(F r m,(X \leftarrow A, Y \leftarrow B, Z \leftarrow C$ else $D)) \in$ match $(D s t s)(($ if $A$ then $B$ else $C$ else $D))$, and $(F r m,(X \leftarrow A$, $Y \leftarrow B$ else $C, Z \leftarrow D)) \notin \operatorname{match}(D$ sts $)(($ if $A$ then $B$ else $C$ else $D))$;

- atom $(D s t s)(E l)=$ true if and only if $E l \in$ id $\cup \bigcup_{\text {Btype } \in \text { btype }} \operatorname{lit}($ Btype).

The form Frm_bool is associated with the type bool, and it is defined as follows: pat $($ Frm_bool $)=(X$ isof bool $)$, pars $($ Frm_bool $)=X$, $\operatorname{vkind}(X)=$ eval, $\operatorname{skind}(X)=$ elem, pcond(Frm_bool) $=$ true, rvcond(Frm_bool) = true, kind(Frm_bool) = statefree, and frmsem $($ Frm_bool $)(E l)=$ true if and only if $E l \in \operatorname{lit}($ bool $)$. 
The form Frm_sort checks whether an element sequence belongs to a particular sort, and it is defined as follows: pat(Frm_sort $)=(X$ is $Y)$, $\operatorname{pars}($ Frm_sort $)=X, \operatorname{vkind}(X)=$ quote, $\operatorname{vkind}(y)=$ eval, $\operatorname{skind}(X)=$ $\operatorname{skind}(Y)=$ seq, pcond(Frm_sort) = true, rvcond(Frm_sort) $=$ ((retval) isof bool), kind(Frm_sort) = statefree, and frmsem(Frm_sort $)(E l)=$ true if and only if $(X$ isof $Y)$. The forms with the pattern $(X$ isof Elseq) for particular sorts Elseq are defined below.

The form Frm_id specifies the characteristic function for $i d$, and it is defined as follows: pat(Frm_id $)=(X$ isof identifier $)$, pars(Frm_id $)=$ $X, \operatorname{vkind}(X)=$ quote, $\operatorname{skind}(X)=$ elem, pcond(Frm_id) $=$ true, rvcond $($ Frm_bool $)=(($ retval $)$ is bool $)$, kind(Frm_id $)=$ statefree, and $\operatorname{frmsem}($ Frm_id $)(E l)=$ true if and only if $E l \in$ id.

The form Frm_btype specifies the characteristic function for btype, and it is defined as follows: pat(Frm_btype $)=(X$ isof btype $)$, $\operatorname{pars}($ Frm_btype $)=X$, $\operatorname{vkind}(X)=$ quote, skind $(X)=$ elem, pcond(Frm_btype $)=(X$ is identifier $)$, rvcond(Frm_btype $)=$ ((retval) is bool), kind(Frm_btype) = statefree, and frmsem(Frm_btype $)(E l)=$ true if and only if $E l \in$ btype.

The form Frm_Btype specifies the characteristic function for Btype $\neq$ bool, and it is defined as follows: pat(Frm_Btype $)=(X$ isof Btype $)$, $\operatorname{pars}($ Frm_Btype $)=X, \operatorname{vkind}(X)=$ eval, $\operatorname{skind}(X)=$ elem, pcond $($ Frm_Btype $)=$ true, rvcond $($ Frm_Btype $)=(($ retval $)$ is bool $)$, kind(Frm_Btype $)=$ statefree, and frmsem(Frm_Btype $)(E l)=$ true if and only if $E l \in \operatorname{lit}($ Btype).

The operations $=$, and $!=$ on basic types, the arithmetic operations,+- , $*$, div, $\bmod$, and arithmetic relations $<,>,<=,>=$ on integers, the boolean operations and, or, not, $\Longrightarrow$ are defined by the corresponding state-free predefined forms Frm_eq, Frm_neq, Frm_add, Frm_sub, Frm_mul, Frm_div, Frm_mod, Frm_less, Frm_more, Frm_lesseq, Frm_moreeq, Frm_and, Frm_or, Frm_not, and Frm_implies:

- $\operatorname{pat}($ Frm_eq $)=(X=Y), \operatorname{pars}($ Frm_eq $)=X Y, \operatorname{vkind}(X)=$ $\operatorname{vkind}(Y \overline{)}=$ eval, $\operatorname{skind}(X)=\operatorname{skind}(Y \overline{)}=$ elem, pcond(Frm_eq $)=$ true, rvcond(Frm_eq) $=(($ retval $)$ is bool $)$, kind(Frm_eq $)=$ statefree, and frmsem(Frm_eq) $\left(E l, E l^{\prime}\right)=$ true if and only if $E l \in$ Btype, $E l^{\prime} \in$ Btype for some Btype, and $E l=E l^{\prime}$;

- $\operatorname{pat}($ Frm add $)=(X+Y), \operatorname{pars}($ Frm add $)=X Y, \operatorname{vkind}(X)=$ $\operatorname{vkind}(Y)=$ eval, $\operatorname{skind}(X)=\operatorname{skind}(Y)=$ elem, pcond(Frm_add $)=$ $((X$ is int $)$ and $(Y$ is int $)), \operatorname{rvcond}(F r m$ add $)=(($ retval $)$ is int $)$, kind $\left(F r m \_a d d\right)=$ statefree, and frmsem $($ Frm_add $)\left(E l, E l^{\prime}\right)=E l^{\prime \prime}$ if and only if $E l^{\prime \prime}=E l+E l^{\prime}$;

- $\operatorname{pat}($ Frm_less $)=(X<Y), \operatorname{pars}($ Frm_less $)=X Y$, $\operatorname{vkind}(X)=$ $\operatorname{vkind}(Y)=$ eval, $\operatorname{skind}(X)=\operatorname{skind}(Y)=$ elem, 
pcond $($ Frm_less $)=((X$ is int $)$ and $(Y$ is int $))$, rvcond(Frm_less $)=(($ retval $)$ is bool $)$, kind(Frm_less $)=$ statefree, and frmsem $($ Frm_less $)\left(E l, E l^{\prime}\right)=$ true if and only if $E l<E l^{\prime}$

- $\operatorname{pat}($ Frm_and $)=(X$ and $Y), \operatorname{pars}($ Frm_and $)=X Y, \operatorname{vkind}(X)=$ $\operatorname{vkind}(Y)=$ eval, $\operatorname{skind}(X)=\operatorname{skind}(Y)=$ elem, pcond(Frm_and $)=$ $((X$ is bool) and $(Y$ is bool $))$, rvcond(Frm_and $)=$ ((retval) is bool), kind(Frm_and) = statefree, and frmsem(Frm_and $)\left(E l, E l^{\prime}\right)=$ true if and only if $E l=$ true, or $E l^{\prime}=$ true.

The other forms are defined in a similar way.

The state $S t$ of Dsts is defined by the forms Frm_isvar, Frm_vartype, and Frm_varval.

The form Frm_isvar specifies which identifiers are variables in $S t$ and is defined as follows: pat(Frm_isvar $)=(X$ is variable $)$, $\operatorname{pars}($ Frm_isvar $)=X, \operatorname{vkind}(X)=$ quote, $\operatorname{skind}(X)=$ elem, pcond(Frm_isvar $)=(X$ is identifier $)$, rvcond(Frm_isvar $)=$ ((retval) is bool), and kind(Frm_isvar) $=$ und.

The form Frm_vartype specifies the types of variables in $S t$ and is defined as follows: pat $($ Frm_vartype $)=($ type of $X)$, pars $($ Frm_vartype $)=$ $X, \operatorname{vkind}(X)=$ quote, $\operatorname{skind}(X)=$ elem, pcond(Frm vartype $)=$ ( $X$ is variable), rvcond(Frm_vartype $)=($ (retval) is btype), and kind(Frm_vartype $)=$ und.

The form Frm_varval specifies the values of variables in $S t$ and is defined as follows: $\operatorname{pat}($ Frm_varval $)=X, \operatorname{pars}($ Frm_varval $)=X, \operatorname{vkind}(X)=$ quote, $\operatorname{skind}(\bar{X})=$ elem, $\quad$ pcond(Frm_varval) $=$ $(X$ is variable $)$, rvcond(Frm_varval $)=(($ retval $)$ is (type of $X))$, and kind(Frm_varval) $=$ und.

The variable declaration is defined by the rule Rul_vardec such that $\operatorname{pat}\left(\operatorname{Rul} \_\operatorname{vardec}\right)=(\operatorname{var} X Y), \operatorname{pars}\left(\operatorname{Rul} \_\operatorname{vardec}\right)=X Y, \operatorname{vkind}(X)=$ $\operatorname{vkind}(Y)=$ quote, $\operatorname{skind}(X)=\operatorname{skind}(Y)=$ elem, pcond $($ Rul vardec $)=$ $((X$ is identifier) and (not $(X$ is btype)) and (not ( $X$ is variable)) and $(Y$ is btype $)$, rvcond(Rul_vardec $)=$ true, rkind(Rul_vardec $)=$ defined, and body (Rul_vardec $)=((X$ is variable $):=$ true $)$ ((type of $X)::=Y)$.

The assignment statement is defined by the rule Rul_assign such that $\operatorname{pat}($ Rul_assign $)=(X:=Y), \operatorname{pars}($ Rul_assign $)=X Y, \operatorname{vkind}(X)=$ quote, $\operatorname{vkind}(Y)=$ eval, $\operatorname{skind}(X)=\operatorname{skind}(Y)=$ elem, pcond(Rul_assign) $=((X$ is variable) and $(Y$ is (type of $X)))$, rvcond(Rul_assign) = true, rkind(Rul_assign) $=$ defined, and body(Rul_assign $)=(X::=Y)$.

The if statement is defined by the rule Rul_if such that pat(Rul_if $)=$ 
(if $X$ then $Y$ else $Z$ ), pars(Rul_if) $=X Y Z$, $\operatorname{vkind}(X)=\operatorname{vkind}(Y)=$ $\operatorname{vkind}(Z)=$ quote, $\operatorname{skind}(X)=$ elem, skind $(Y)=\operatorname{skind}(Z)=$ seq, pcond(Rul_if $)=(X$ is bool), rvcond(Rul_if $)=$ true, rkind(Rul_if $)=$ defined, and body $($ Rul_if $)=(\operatorname{cases}($ if $X$ then $Y)$ $($ else $Z)$ ).

The while statement is defined by the rule Rul_while such that $\operatorname{pat}($ Rul_while $)=($ while $X$ do $Y$ ), pars(Rul_while) $=X Y$, $\operatorname{vkind}(X)=\operatorname{vkind}(Y)=$ quote, $\operatorname{skind}(X)=$ elem, $\operatorname{skind}(Y)=$ seq, pcond(Rul_while $)=$ true, $\operatorname{rvcond}(\mathrm{Rul}$ while $)=$ true, rkind(Rul_while $)=$ defined, and body(Rul_while) $=(\operatorname{cases}($ if $X$ then $Y$ (while $X$ do $Y)$ ) (else)).

An element sequence is called a program in the MPL language. A program Elseq is called correct in St, if Elseq is a correct sequence in St. A program Elseq is called incorrect in $S t$ if Elseq is not correct in $S t$.

The program (var $X$ int) $(X:=5)$ (if $(X=5)$ then $(X:=$ $0)$ else) is correct in the empty state. Its execution returns the state $S t^{\prime}$ such that $\operatorname{dom}\left(S t^{\prime}\right)=\{$ Frm_isvar, Frm_vartype, Frm_varval $\}, \operatorname{graph}\left(S t^{\prime}(\right.$ Frm_isvar $))=\{(X$, true $)\}, \operatorname{graph}\left(S \bar{t}^{\prime}(\right.$ Frm_vartype $\left.)\right)=\{(X$, int $)\}$, and $\operatorname{graph}\left(S t^{\prime}(\right.$ Frm_varval $\left.)\right)=\{(X, 0)\}$.

The program $(X:=5)$ is incorrect in the empty state, since in accordance with the definition of the rule Rul_assign the identifier $X$ must be a variable in this state.

The program (assume $((X$ is variable) and ((type of $X)$ is int $)))$ $(X:=5)$ is correct in the empty statement, since in accordance with the definition of assume the assignment $(X:=5)$ will not be executed. In accordance with the definition of the controlled backtracking, execution of this program terminates in the empty statement.

The program (var $X$ int) $(X:=5)$ (var $X$ int) is incorrect in the empty statement, since in accordance with the definition of the rule Rul_vardec a variable can not be declared twice.

\section{Conclusion}

DSTSs are a special type of transition systems for determining domainspecific languages used to solve the problems of the development of computer language semantics and of the design, specification, prototyping, and verification of software systems. DSTSs form the basis of a comprehensive approach to solving these problems.

In this paper, the new object model of DSTSs has been described. It introduces new entities and concepts into the theory of DSTSs such as forms, element generators, and propagation of the indeterminate value with its handling. It also extends the concepts of substitution and pattern matching, determines the classification of forms and transition rules, adds constraints 
on the parameters and the return values of forms, improves the algorithm for finding the element values, considers the transition rules as a special kind of forms, improves the definitions of backtracking, safe configurations and runs, and correct control element sequences. The formal definition of the model programming language with the extensible set of basic types, based on this model, has been also given.

\section{References}

[1] Anureev I.S. Domain-specific transition systems: object model and language // System Informatics. - 2013. - No. 1. - P. 1-34 (In Russian).

[2] Anureev I.S. Integrated approach to analysis and verification of imperative programs // Bulletin NCC. Series: Computer Science. - Novosibirsk, 2011.-IIS Special Iss. 32. - P. 1-18.

[3] Anureev I.S. Introduction to the Atoment language // Bulletin NCC. Series: Computer Science. - Novosibirsk, 2010. - IIS Special Iss. 31. - P. 1-16.

[4] Anureev I.S. Typical examples of using the Atoment language // Automatic Control and Computer Sciences. - 2012. - Vol. 46, No. 7. - P. 299-307.

[5] Anureev I.S., Maryasov I.V., Nepomniaschy V.A. Two-level mixed verification method of C-light programs in terms of safety logic // Bulletin NCC. Series: Computer Science. - Novosibirsk, 2012. - IIS Special Iss. 34. - P. 23-42.

[6] Anureev I.S. Program specific transition systems // Bulletin NCC. Series: Computer Science. - Novosibirsk, 2012. - IIS Special Iss. 34. - P. 1-21.

[7] AsmL: The Abstract state Machine Language. Reference Manual, 2002. http://research.microsoft.com/en-us/projects/asml/

[8] Gurevich Y. Abstract state Machines: An Overview of the Project // Foundations of Information and Knowledge Systems (FoIKS). Proc. Third Internat. Symp. - Lect. Notes Comput. Sci. - 2004. - Vol. 2942. - P. 6-13.

[9] Anlauff M. XasM - An Extensible, Component-Based Abstract State Machines Language. -

http://xasm.sourceforge.net/XasmAnl00/XasmAnl00.html

[10] Nepomniaschy V.A., Anureev I.S., Atuchin M.M., Maryasov I.V., Petrov A.A., Promsky A.V. C program verification in Spectrum multilanguage system // Automatic Control and Computer Sciences. - 2011. - Vol. 45, No. 7. P. 413-420.

[11] Parnas D.L. Really rethinking formal methods // Computer. IEEE Computer Society. - 2010. - Vol. 43 (1). - P. 28-34. 
